\title{
Anharmonic Oscillators with Infinitely Many Real Eigenvalues and $\mathcal{P} \mathcal{T}$-Symmetry ${ }^{\star}$
}

Kwang C. SHIN

Department of Mathematics, University of West Georgia, Carrollton, GA, 30118, USA

E-mail: kshin@westga.edu

Received October 11, 2009, in final form January 28, 2010; Published online February 03, 2010

doi:10.3842/SIGMA.2010.015

\begin{abstract}
We study the eigenvalue problem $-u^{\prime \prime}+V(z) u=\lambda u$ in the complex plane with the boundary condition that $u(z)$ decays to zero as $z$ tends to infinity along the two rays $\arg z=-\frac{\pi}{2} \pm \frac{2 \pi}{m+2}$, where $V(z)=-(i z)^{m}-P(i z)$ for complex-valued polynomials $P$ of degree at most $m-1 \geq 2$. We provide an asymptotic formula for eigenvalues and a necessary and sufficient condition for the anharmonic oscillator to have infinitely many real eigenvalues.
\end{abstract}

Key words: anharmonic oscillators; asymptotic formula; infinitely many real eigenvalues; $\mathcal{P} \mathcal{T}$-symmetry

2010 Mathematics Subject Classification: 34L20; 34L40

\section{Introduction}

Theory of the so-called $\mathcal{P} \mathcal{T}$-symmetric Hamiltonians has been a very active research area in recent years $[1,2,3,5,7,8,9,10,13]$. While they are not self-adjoint in general, many $\mathcal{P} \mathcal{T}$ symmetric Hamiltonians have real spectra [1, 3, 5, 9]. Self-adjointness of Hamiltonians is not physical requirement, but guarantees reality of spectrum and conservation of probability. So one searches for non-self-adjoint Hamiltonians that have real spectra. In this paper, we study the Schrödinger eigenvalue problems in the complex plane with complex polynomial potentials under decaying boundary conditions along two fixed rays to infinity. We will prove that the Schrödinger eigenvalue problem has infinitely many real eigenvalues if and only if the eigenvalue problem is $\mathcal{P} \mathcal{T}$-symmetric or it is a translation of a $\mathcal{P} \mathcal{T}$-symmetric problem.

For an integer $m \geq 3$ we consider the Schrödinger eigenvalue problem

$$
H u(z):=\left[-\frac{d^{2}}{d z^{2}}-(i z)^{m}-P(i z)\right] u(z)=\lambda u(z), \quad \text { for some } \lambda \in \mathbb{C},
$$

with the boundary condition that

$$
u(z) \rightarrow 0 \text { exponentially, as } z \rightarrow \infty \text { along the two rays } \arg (z)=-\frac{\pi}{2} \pm \frac{2 \pi}{m+2},
$$

where $P$ is a polynomial of degree at most $m-1$ of the form

$$
P(z)=a_{1} z^{m-1}+a_{2} z^{m-2}+\cdots+a_{m}, \quad a:=\left(a_{1}, a_{2}, \ldots, a_{m}\right) \in \mathbb{C}^{m} .
$$

The existence of infinitely many eigenvalues is known. Sibuya [12] showed that the eigenvalues of $H$ are zeros of an entire function of order $\rho:=\frac{1}{2}+\frac{1}{m} \in(0,1)$ and hence, by the Hadamard factorization theorem (see, e.g., [4]), there are infinitely many eigenvalues.

${ }^{\star}$ This paper is a contribution to the Proceedings of the 5-th Microconference "Analytic and Algebraic Methods V". The full collection is available at http://www.emis.de/journals/SIGMA/Prague2009.html 
The Hamiltonian $H$ in (1) with the potential $V(z)$ under the boundary condition (2) is called $\mathcal{P} \mathcal{T}$-symmetric if $\overline{V(-\bar{z})}=V(z), z \in \mathbb{C}$. Note that $V(z)=-(i z)^{m}-P(i z)$ is a $\mathcal{P} \mathcal{T}$-symmetric potential if and only if $a \in \mathbb{R}^{m}$.

In this paper, we will derive the following asymptotic expansion of the eigenvalues $\lambda_{n}$, ordered in the order of their magnitude.

Theorem 1. There exists $N=N(m) \in \mathbb{Z}$ such that the eigenvalues $\left\{\lambda_{n}\right\}_{n=N}^{\infty}$ of $H$ satisfy that

$$
\sum_{j=0}^{m+1} c_{j}(a) \lambda_{n}^{\rho-\frac{j}{m}}+O\left(\lambda_{n}^{-\rho}\right)=n+\frac{1}{2} \quad \text { as } n \rightarrow+\infty,
$$

where $c_{0}(a)=\pi^{-1} \sin \left(\frac{\pi}{m}\right) B\left(\frac{1}{2}, 1+\frac{1}{m}\right)$ and $c_{1}(a)=0$ and $c_{j}(a), 2 \leq j \leq m+1$, are explicit non-zero polynomials in the coefficients a of the polynomial potential, defined in (18).

As a consequence of (3), we obtain a necessary and sufficient condition for the anharmonic oscillator $H$ to have infinitely many real eigenvalues as follows.

Theorem 2. The anharmonic oscillator $H$ with a potential $V(z)$ has infinitely many real eigenvalues if and only if $H$ with the potential $V\left(z-z_{0}\right)$ is $\mathcal{P} \mathcal{T}$-symmetric for some $z_{0} \in \mathbb{C}$.

Moreover, no $H$ has infinitely many real and infinitely many non-real eigenvalues.

If $H$ is $\mathcal{P} \mathcal{T}$-symmetric, then $u(z)$ is an eigenfunction associated with the eigenvalue $\lambda$ if and only if $\overline{u(-\bar{z})}$ is an eigenfunction associated with the eigenvalue $\bar{\lambda}$. Thus, the eigenvalues of a $\mathcal{P} \mathcal{T}$-symmetric Hamiltonian $H$ either are real or come in complex conjugates. Also, magnitude of the large eigenvalues is strictly increasing [10]. Thus, if $H$ is $\mathcal{P} \mathcal{T}$-symmetric, then eigenvalues are all real with at most finitely many exceptions.

This paper is organized as follows. In Section 2, we will introduce results of Hille [6], Sibuya [12], and Shin [10,11] on properties of the solutions of (1). We will present asymptotics of solutions of (1), introduce the spectral determinant of $H$, and provide an asymptotic expansion for the spectral determinant in the sector where all but finitely many eigenvalues lie. In Section 3, we will prove Theorem 1 where we use the asymptotic expansion of the spectral determinant. In Section 4, we will prove Theorem 2 and provide other spectral results; expres$\operatorname{sing} \lambda_{n}$ as an asymptotic formula in $n$ and obtaining an asymptotic formula for nearest neighbor spacing of eigenvalues, monotonicity of their magnitude, and more.

\section{Properties of the solutions}

In this section, we will introduce results of Hille [6], Sibuya [12], and Shin [10, 11] on the properties of solutions of (1).

First, let $u$ be a solution of (1) and let $v(z)=u(-i z)$. Then $v$ solves

$$
-v^{\prime \prime}(z)+\left[z^{m}+P(z)+\lambda\right] v(z)=0 .
$$

Solutions of (4) have simple asymptotic behavior near infinity in the complex plane and in order to describe the asymptotic behavior, we will use the Stokes sectors. The Stokes sectors $S_{k}$ of the equation (4) are

$$
S_{k}=\left\{z \in \mathbb{C}:\left|\arg (z)-\frac{2 k \pi}{m+2}\right|<\frac{\pi}{m+2}\right\} \quad \text { for } k \in \mathbb{Z} .
$$

Hille $[6, \S 7.4]$ showed that every nonconstant solution of (4) either decays to zero or blows up exponentially in each Stokes sector. Moreover, if a nonconstant solution $v$ of (4) decays in 
a Stokes sector, it must blow up in its two adjacent Stokes sectors. However, when $v$ blows up in a Stokes sector, $v$ need not be decaying in its adjacent Stokes sectors. Thus, the decaying boundary condition (2) becomes that $v(z) \rightarrow 0$ as $z \rightarrow \infty$ in the sectors $S_{1} \cup S_{-1}$.

We will also use the following functions of the coefficients $a \in \mathbb{C}^{m}$ of the polynomial $P$. Let

$$
\begin{aligned}
& b_{j, k}(a) \text { be the coefficient of } z^{m k-j} \text { in }\left(\begin{array}{c}
\frac{1}{2} \\
k
\end{array}\right)(P(z))^{k} \text { for } 1 \leq k \leq j, \text { and } \\
& b_{j}(a)=\sum_{k=1}^{j} b_{j, k}(a), \quad j \in \mathbb{N} .
\end{aligned}
$$

We also let $r_{m}=-\frac{m}{4}-\nu(a)$ and $\mu(a)=\frac{m}{4}-\nu(a)$, where

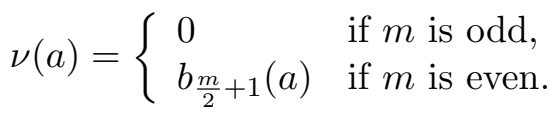

Now we are ready to introduce results of Sibuya [12] and Shin [11] on existence of a solution that has a fixed asymptotic behavior in $S_{-1} \cup S_{0} \cup S_{1}$, and on its properties.

Theorem 3. Equation (4), with $a \in \mathbb{C}^{m}$, has a solution $f(z, a, \lambda)$ with the following properties.

(i) $f(z, a, \lambda)$ is an entire function of $z, a$ and $\lambda$.

(ii) $f(z, a, \lambda)$ and $f^{\prime}(z, a, \lambda)=\frac{\partial}{\partial z} f(z, a, \lambda)$ admit the following asymptotic expansions. For each fixed $\varepsilon>0$,

$$
\begin{aligned}
& f(z, a, \lambda)=z^{r_{m}}\left(1+O\left(z^{-1 / 2}\right)\right) \exp [-F(z, a, \lambda)], \\
& f^{\prime}(z, a, \lambda)=-z^{r_{m}+\frac{m}{2}}\left(1+O\left(z^{-1 / 2}\right)\right) \exp [-F(z, a, \lambda)],
\end{aligned}
$$

as $z$ tends to infinity in the sector $|\arg z| \leq \frac{3 \pi}{m+2}-\varepsilon$, uniformly on each compact set of $(a, \lambda)$-values. Here

$$
F(z, a, \lambda)=\frac{2}{m+2} z^{\frac{m}{2}+1}+\sum_{1 \leq j<\frac{m}{2}+1} \frac{2}{m+2-2 j} b_{j}(a) z^{\frac{1}{2}(m+2-2 j)} .
$$

(iii) For each $\delta>0, f$ and $f^{\prime}$ also admit the asymptotic expansions,

$$
\begin{aligned}
& f(0, a, \lambda)=\left[1+O\left(\lambda^{-\rho}\right)\right] \lambda^{-1 / 4} \exp [L(a, \lambda)], \\
& f^{\prime}(0, a, \lambda)=-\left[1+O\left(\lambda^{-\rho}\right)\right] \lambda^{1 / 4} \exp [L(a, \lambda)],
\end{aligned}
$$

as $\lambda \rightarrow \infty$ in the sector $|\arg (\lambda)| \leq \pi-\delta$, uniformly on each compact set of $a \in \mathbb{C}^{m}$, where $\rho:=\frac{1}{2}+\frac{1}{m}$

$$
L(a, \lambda)=\int_{0}^{+\infty}\left(\sqrt{t^{m}+P(t)+\lambda}-t^{\frac{m}{2}}-\sum_{j=1}^{\left\lfloor\frac{m+1}{2}\right\rfloor} b_{j}(a) t^{\frac{m}{2}-j}-\frac{\nu(a)}{t+1}\right) d t .
$$

(iv) The entire functions $\lambda \mapsto f(0, a, \lambda)$ and $\lambda \mapsto f^{\prime}(0, a, \lambda)$ have orders $\rho=\frac{1}{2}+\frac{1}{m}$.

Proof. See Theorems 6.1, 7.2, 19.1, and 20.1 in [12] for proof. Sibuya showed (5) and (6) with error terms $o(1)$ and I improved them to $O\left(\lambda^{-\rho}\right)$ in [11]. 


\section{$2.1 \quad$ Spectral determinant}

In this subsection, we will introduce the function whose zeros are the eigenvalues of $H$.

Let

$$
\omega=\exp \left[\frac{2 \pi i}{m+2}\right]
$$

and define

$$
G^{k}(a):=\left(\omega^{-k} a_{1}, \omega^{-2 k} a_{2}, \ldots, \omega^{-m k} a_{m}\right) \quad \text { for } k \in \mathbb{Z} .
$$

Then one can see from the definition of $b_{j, k}(a)$ that

$$
b_{j, k}\left(G^{\ell}(a)\right)=\omega^{-j \ell} b_{j, k}(a) \quad \text { and } \quad b_{j}\left(G^{\ell}(a)\right)=\omega^{-j \ell} b_{j}(a) \quad \text { for } \ell \in \mathbb{Z} .
$$

Also, by Theorem 3 there exists $f\left(z, G^{k}(a), \omega^{-m k} \lambda\right)$ that decays in $S_{0}$ and we let

$$
f_{k}(z, a, \lambda):=f\left(\omega^{-k} z, G^{k}(a), \omega^{-m k} \lambda\right) .
$$

So $f_{k}$ decays in $S_{k}$ and blows up in its two adjacent Stokes sectors. Since $f_{0}$ decays in $S_{0}$ while $f_{1}$ blows up in the same sector, we conclude that $f_{0}$ and $f_{1}$ are linearly independent. Thus, any solution can be expressed as a linear combination of these two. In particular,

$$
f_{-1}(z, a, \lambda)=C(a, \lambda) f_{0}(z, a, \lambda)+\widetilde{C}(a, \lambda) f_{1}(z, a, \lambda) \text { for some } C(a, \lambda) \text { and } \widetilde{C}(a, \lambda) .
$$

One can write these coefficients in terms of the Wronskians of solutions. That is,

$$
C(a, \lambda)=\frac{\mathcal{W}_{-1,1}(a, \lambda)}{\mathcal{W}_{0,1}(a, \lambda)} \quad \text { and } \quad \widetilde{C}(a, \lambda)=-\frac{\mathcal{W}_{-1,0}(a, \lambda)}{\mathcal{W}_{0,1}(a, \lambda)},
$$

where $\mathcal{W}_{j, k}=f_{j} f_{k}^{\prime}-f_{j}^{\prime} f_{k}$ is the Wronskian of $f_{j}$ and $f_{k}$. Since $f_{0}$ and $f_{1}$ are linearly independent, $\mathcal{W}_{0,1}(a, \lambda) \neq 0$ and likewise $\mathcal{W}_{-1,0}(a, \lambda) \neq 0$. Then by Theorem $3(i)$, for each fixed $a \in \mathbb{C}^{m}$, the function $\lambda \mapsto C(a, \lambda)$ is entire. Moreover, $\lambda$ is an eigenvalue of $H$ if and only if $C(a, \lambda)=0$. We call $C(a, \lambda)$ the spectral determinant of $H$.

\section{$2.2 \quad$ Asymptotic expansions of $C(a, \lambda)$}

In [10], the asymptotics of $C(a, \lambda)$ is provided and it is showed that all but finitely many zeros $\lambda$ of $C(a, \lambda)$ lie in a small sector containing the positive real $\lambda$-axis. Here we will improve this asymptotics of $C(a, \lambda)$ as $\lambda \rightarrow \infty$ in a sector containing the positive real $\lambda$-axis as follows.

Theorem 4. Suppose that $m \geq 4$. Then for each fixed $a \in \mathbb{C}^{m}$ and $0<\delta<\frac{\pi}{m+2}$,

$$
\begin{aligned}
C(a, \lambda)= & {\left[\omega^{\frac{1}{2}}+O\left(\lambda^{-\rho}\right)\right] \exp \left[L\left(G^{-1}(a), \omega^{-2} \lambda\right)-L(a, \lambda)\right] } \\
& +\left[\omega^{\frac{1}{2}+2 \nu(a)}+O\left(\lambda^{-\rho}\right)\right] \exp \left[L\left(G(a), \omega^{2} \lambda\right)-L(a, \lambda)\right],
\end{aligned}
$$

as $\lambda \rightarrow \infty$ in the sector

$$
\pi-\frac{4\left\lfloor\frac{m}{2}\right\rfloor \pi}{m+2}+\delta \leq \arg (\lambda) \leq \pi-\frac{4 \pi}{m+2}-\delta .
$$

If $m=3$ then

$$
\begin{aligned}
C(a, \lambda)= & {\left[-\omega^{-2}+O\left(\lambda^{-\rho}\right)\right] \exp \left[L\left(G^{4}(a), \omega^{-2} \lambda\right)-L(a, \lambda)\right] } \\
& -\left[i \omega^{\frac{7}{4}}+O\left(\lambda^{-\rho}\right)\right] \exp \left[-L\left(G^{2}(a), \omega^{-1} \lambda\right)-L(a, \lambda)\right],
\end{aligned}
$$

as $\lambda \rightarrow \infty$ in the sector

$$
-\frac{\pi}{5}+\delta \leq \arg (\lambda) \leq \pi-\delta
$$


Proof. In Theorems 13 and 14 of Shin [10], similar asymptotics of $C(a, \lambda)$ are proved with the error terms $o(1)$ instead of $O\left(\lambda^{-\rho}\right)$. With the improved asymptotics (5) and (6), one can closely follow proofs of Theorems 13 and 14 in [10] to complete the proof.

Next, in order to further examine the improved asymptotics of $C(a, \lambda)$ in Theorem 4 , we will use the following asymptotics of $L(a, \lambda)$.

Lemma 1. Let $m \geq 3$ and $a \in \mathbb{C}^{m}$ be fixed. Then

$$
L(a, \lambda)=\sum_{j=0}^{\infty} K_{m, j}(a) \lambda^{\frac{1}{2}+\frac{1-j}{m}}-\frac{\nu(a)}{m} \ln (\lambda)
$$

as $\lambda \rightarrow \infty$ in the sector $|\arg (\lambda)| \leq \pi-\delta$ with $K_{m, 0}(a)=\frac{B\left(\frac{1}{2}, 1+\frac{1}{m}\right)}{2 \cos \left(\frac{\pi}{m}\right)}>0$ and for $j \geq 1$,

$$
K_{m, j}(a)=\sum_{k=\left\lfloor\frac{j-1}{m}\right\rfloor+1}^{j} K_{m, j, k} b_{j, k}(a),
$$

where for $\frac{j}{m} \leq k \leq j$,

$$
K_{m, j, k}:= \begin{cases}-\frac{2}{m} & \text { if } j=1, \\ \frac{2}{m}\left(\ln 2-\sum_{s=1}^{k-1} \frac{1}{2 s-1}\right) & \text { if } j=\frac{m}{2}+1, m \text { even, } \\ \frac{1}{m} B\left(k-\frac{j-1}{m}, \frac{j-1}{m}-\frac{1}{2}\right) & \text { if } j \neq 1 \text { or } j \neq \frac{m}{2}+1 .\end{cases}
$$

Proof. See the Appendix in [11] for a proof.

Remark 1. The coefficients $K_{m, j}(a)$ have the following properties that can be deduced from (12) and the definition of $b_{j, k}(a)$ :

(i) The $K_{m, j}(a)$ are all real polynomials in terms of the coefficients $a$ of $P$.

(ii) For each $1 \leq j \leq m$, the polynomial $K_{m, j}(a)$ depends only on $a_{1}, a_{2}, \ldots, a_{j}$. Furthermore, it is a non-constant linear function of $a_{j}$.

\section{Proof of Theorem 1}

In this section, we will prove Theorem 1.

Proof of Theorem 1. In order to get asymptotics for the eigenvalues $\lambda$, we examine asymptotics of $C(a, \lambda)$ since the zeros of $C(a, \lambda)$ are the eigenvalues of $H$. Since all but finitely many eigenvalues lie near the positive real $\lambda$-axis, the asymptotics in Theorem 4 would be sufficient for our purpose.

For $m \geq 4$ and $a \in \mathbb{C}^{m}$ fixed, we set $C(a, \lambda)=0$ in (8), rearrange the asymptotic equation to get

$$
\left[1+O\left(\lambda^{-\rho}\right)\right] \exp \left[L\left(G(a), \omega^{2} \lambda\right)-L\left(G^{-1}(a), \omega^{-2} \lambda\right)\right]=-\omega^{-2 \nu(a)},
$$

and absorb the term $\left[1+O\left(\lambda^{-\rho}\right)\right]$ into the exponential term to obtain

$$
\exp \left[L\left(G(a), \omega^{2} \lambda\right)-L\left(G^{-1}(a), \omega^{-2} \lambda\right)+O\left(\lambda^{-\rho}\right)\right]=-\omega^{-2 \nu(a)},
$$


or equivalently

$$
L\left(G(a), \omega^{2} \lambda\right)-L\left(G^{-1}(a), \omega^{-2} \lambda\right)+O\left(\lambda^{-\rho}\right)=(2 n+1) \pi i-\frac{4 \nu(a) \pi}{m+2} i, \quad \text { for some } n \in \mathbb{Z} .
$$

Since $L(a, \lambda)=K_{m, 0}(a) \lambda^{\rho}(1+o(1))$ by Lemma 1 , one gets

$$
L\left(G(a), \omega^{2} \lambda\right)-L\left(G^{-1}(a), \omega^{-2} \lambda\right)+O\left(\lambda^{-\rho}\right)=2 i K_{m, 0}(a) \sin \left(\frac{2 \pi}{m}\right) \lambda^{\rho}(1+o(1)),
$$

as $\lambda \rightarrow \infty$ in the sector (9) containing the positive real $\lambda$-axis. Thus, the function

$$
\lambda \mapsto H(a, \lambda):=L\left(G(a), \omega^{2} \lambda\right)-L\left(G^{-1}(a), \omega^{-2} \lambda\right)+O\left(\lambda^{-\rho}\right)
$$

maps the region $|\lambda| \geq M$ and $|\arg (\lambda)| \leq \varepsilon$ for some large $M>0$ and small $\varepsilon>0$ onto a region that contains the positive imaginary axis near infinity. Also, $H(a, \cdot)$ is analytic in the region $|\lambda| \geq M$ and $|\arg (\lambda)| \leq \varepsilon$. Then, Sibuya [12, pp. 131-133] showed that for every closed ball $\left\{a \in \mathbb{C}^{m}:|a| \leq r\right\}$, there exists $N_{0}=N_{0}(m, r) \in \mathbb{N}$ such that for every integer $n \geq N_{0}$ there exists exactly one $\lambda_{n}$ for which (13) holds, that is,

$$
H\left(a, \lambda_{n}\right)=(2 n+1) \pi i-\frac{4 \nu(a) \pi}{m+2} i .
$$

(Sibuya showed the existence of such an $N_{0}$ with the estimate (14).)

Hence, from Lemma 1 we infer that

$$
\begin{aligned}
H\left(a, \lambda_{n}\right)= & L\left(G(a), \omega^{2} \lambda_{n}\right)-L\left(G^{-1}(a), \omega^{-2} \lambda_{n}\right)+O\left(\lambda_{n}^{-\rho}\right) \\
= & \sum_{j=0}^{m+1}\left[K_{m, j}(G(a))\left(\omega^{2} \lambda_{n}\right)^{\frac{1}{2}+\frac{1-j}{m}}-K_{m, j}\left(G^{-1}(a)\right)\left(\omega^{-2} \lambda_{n}\right)^{\frac{1}{2}+\frac{1-j}{m}}\right] \\
& +\frac{\nu\left(G^{-1}(a)\right)}{m} \ln \left(\omega^{-2} \lambda_{n}\right)-\frac{\nu(G(a))}{m} \ln \left(\omega^{2} \lambda_{n}\right)+O\left(\lambda_{n}^{-\rho}\right) \\
= & 2 i \sum_{j=0}^{m+1} K_{m, j}(a) \sin \left(\frac{2(1-j) \pi}{m}\right) \lambda_{n}^{\frac{1}{2}+\frac{1-j}{m}}+\frac{\nu(a)}{m} \frac{8 \pi i}{m+2}+O\left(\lambda_{n}^{-\rho}\right),
\end{aligned}
$$

where we used $\nu\left(G^{ \pm 1}(a)\right)=-\nu(a)$ and by (7) and (12), for $1 \leq j \leq m+1$,

$$
\begin{aligned}
K_{m, j} & (G(a))\left(\omega^{2}\right)^{\frac{1}{2}+\frac{1-j}{m}}-K_{m, j}\left(G^{-1}(a)\right)\left(\omega^{-2}\right)^{\frac{1}{2}+\frac{1-j}{m}} \\
& =K_{m, j}(a) \omega^{-j}\left(\omega^{2}\right)^{\frac{1}{2}+\frac{1-j}{m}}-K_{m, j}(a) \omega^{j}\left(\omega^{-2}\right)^{\frac{1}{2}+\frac{1-j}{m}} \\
& =K_{m, j}(a)\left(e^{(1-j) \frac{2 \pi i}{m}}-e^{-(1-j) \frac{2 \pi i}{m}}\right) \\
& =2 i K_{m, j}(a) \sin \left(\frac{2(1-j) \pi}{m}\right) .
\end{aligned}
$$

Next, one can combine (15) and (16) and rearrange the resulting equation to obtain (3). That is,

$$
\sum_{j=0}^{m+1} c_{j}(a) \lambda_{n}^{\frac{1}{2}+\frac{1-j}{m}}+O\left(\lambda_{n}^{-\rho}\right)=n+\frac{1}{2} \quad \text { as } n \rightarrow \infty,
$$

where for $0 \leq j \leq m+1$,

$$
c_{j}(a):= \begin{cases}\frac{1}{\pi} K_{m, j}(a) \sin \left(\frac{2(1-j) \pi}{m}\right) & \text { if } j \neq \frac{m}{2}+1, \\ \frac{2 \nu(a)}{m} & \text { if } j=\frac{m}{2}+1, m \text { even. }\end{cases}
$$


We still need to examine how many eigenvalues of $H$ are not in the set $\left\{\lambda_{n}\right\}_{n \geq N_{0}}$ to complete our proof. To do this we will use Hurwitz's theorem in complex analysis.

Hurwitz's theorem [4] says that if a sequence of analytic functions $\varphi_{k}$ converges uniformly to an analytic function $\varphi$ on any compact subsets of their common domain, then for all large $k$ functions $\varphi_{k}$ and $\varphi$ have the same number of zeros in any open subset whose boundary does not contain any zeros of $\varphi$. Since the eigenvalues are the zeros of $C(a, \lambda)$ that is an analytic function in variables $a$ and $\lambda$, Hurwitz's theorem implies that eigenvalues varies continuously as $a$ varies. That is, $\lambda_{n}(a)$ varies continuously from $\lambda_{n}(\widehat{a})$ to $\lambda_{n}(\widetilde{a})$ as $a$ varies from $\widehat{a}$ to $\widetilde{a}$ in the closed ball, where we used $\lambda_{n}(a)$ in order to clearly indicate its dependence on $a$.

When $a=0$, there exists an integer $N=N(m) \leq N_{0}$ such that the eigenvalues of $H$ that are not in $\left\{\lambda_{n}(0)\right\}_{n \geq N_{0}}$ is exactly $\left(N_{0}-N\right)$. Also, Hurwitz's theorem implies that when eigenvalues collide as $a$ varies, the number of eigenvalues before and after the collision remains the same. So there is no sudden appearance or disappearance of the eigenvalues as $a$ varies and hence, there are exactly $\left(N_{0}-N\right)$-eigenvalues that are not in the set $\left\{\lambda_{n}(a)\right\}_{n \geq N_{0}}$. Therefore, the eigenvalues $\left\{\lambda_{n}(a)\right\}_{n \geq N}$ satisfy (3). This completes the proof for $m \geq 4$.

For $m=3$, proof is very similar to the case $m \geq 4$ above and we will use (10) in the place of (8). If $C(a, \lambda)=0$ then we rearrange the asymptotic formula (10) with $C(a, \lambda)=0$ to obtain

$$
\left[1+O\left(\lambda^{-\rho}\right)\right] \exp \left[-L\left(G^{4}(a), \omega^{-2} \lambda\right)-L\left(G^{2}(a), \omega^{-1} \lambda\right)\right]=e^{\pi i},
$$

as $\lambda \rightarrow \infty$ in the sector (11). Next, like in (17) we examine

$$
\begin{aligned}
-K_{3, j} & \left(G^{4}(a)\right)\left(\omega^{-2} \lambda\right)^{\frac{1}{2}+\frac{1-j}{3}}-K_{3, j}\left(G^{2}(a)\right)\left(\omega^{-1} \lambda\right)^{\frac{1}{2}+\frac{1-j}{3}} \\
& =-\left[\omega^{-4 j} \omega^{-2\left(\frac{1}{2}+\frac{1-j}{3}\right)}+\omega^{-2 j} \omega^{-\left(\frac{1}{2}+\frac{1-j}{3}\right)}\right] K_{3, j}(a) \lambda^{\frac{1}{2}+\frac{1-j}{3}} \\
& =-\left[e^{-\frac{4(j-1)}{3} \pi i}-e^{-\frac{2(j-1)}{3} \pi i}\right] K_{3, j}(a) \lambda^{\frac{1}{2}+\frac{1-j}{3}} \\
& =2 i \sin \left(\frac{2(1-j) \pi}{3}\right) K_{3, j}(a) \lambda^{\frac{1}{2}+\frac{1-j}{3}}
\end{aligned}
$$

where we used (7), (12), and $\omega^{\frac{5}{2}}=-1$. Then one can complete proof for $m=3$ just like in the case of $m \geq 4$.

Remark 2. We order the eigenvalues in the order of their magnitude and we showed that eigenvalues vary continuously as the coefficients $a=\left(a_{1}, \ldots, a_{m}\right)$ vary. For the large eigenvalues, $\lambda_{n}(a)$ are continuous functions of $a \in \mathbb{C}^{m}$ (c.f. (20)). However, for small eigenvalues, even though eigenvalues varies continuously as $a$ varies, we do not claim that $\lambda_{n}(a)$ ordered in their order of magnitude are continuous. While these small eigenvalues are continuously moving in the complex plane, their indices can be switched and how we number these small eigenvalues does not affect the asymptotic result in Theorem 1.

\section{Corollaries and proof of Theorem 2}

In this section, we will introduce some results deduced from Theorem 1.

First, we invert (3), expressing $\lambda_{n}$ as an asymptotic formula in $n$. Also, we obtain an asymptotic formula for nearest neighbor spacing of eigenvalues and monotonicity of their magnitude.

Corollary 1. One can compute numbers $d_{j}(a)$ explicitly such that

$$
\lambda_{n}=\sum_{j=0}^{m+1} d_{j}(a) \cdot\left(n+\frac{1}{2}\right)^{\frac{2 m}{m+2}\left(1-\frac{j}{m}\right)}+O\left(n^{\left.-\frac{4}{m+2}\right)} \quad \text { as } n \rightarrow+\infty .\right.
$$


Also, the space between successive eigenvalues is

$$
\lambda_{n+1}-\lambda_{n} \underset{n \rightarrow+\infty}{=} \frac{2 m}{m+2}\left(\frac{\pi}{B\left(\frac{1}{2}, 1+\frac{1}{m}\right)}\right)^{\frac{2 m}{m+2}} \cdot\left(n+\frac{1}{2}\right)^{\frac{m-2}{m+2}}+o\left(n^{\frac{m-2}{m+2}}\right) \quad \text { as } n \rightarrow+\infty .
$$

In particular, $\lim _{n \rightarrow \infty}\left|\lambda_{n+1}-\lambda_{n}\right|=\infty$ and $\lim _{n \rightarrow+\infty} \arg \left(\lambda_{n}\right)=0$. Hence:

$$
\left|\lambda_{n}\right|<\left|\lambda_{n+1}\right| \text { for all large } n \text {. }
$$

Proof. Since (3) is an asymptotic equation, one can solve it for $\lambda_{n}$ to get (19), where $d_{0}(a)=$ $\left(c_{0}\right)^{-\frac{2 m}{m+2}}$ and $d_{j}(a)$ for $1 \leq j \leq m+1$ can be computed recursively and explicitly. Then since $d_{0}>0$, one can deduce

$$
\lim _{n \rightarrow 0} \arg \left(\lambda_{n}\right)=0 \quad \text { and } \quad \lim _{n \rightarrow \infty}\left|\lambda_{n}\right|=\infty .
$$

Also, we obtain

$$
\lambda_{n+1} \underset{n \rightarrow+\infty}{=} \lambda_{n}+\frac{2 m}{m+2}\left(d_{0}(a)\right)^{\frac{2 m}{m+2}} \cdot\left(n+\frac{1}{2}\right)^{\frac{m-2}{m+2}}+o\left(n^{\frac{m-2}{m+2}}\right) \quad \text { as } n \rightarrow+\infty,
$$

where we use the generalized binomial expansion to $\left(1+\left(n+\frac{1}{2}\right)^{-1}\right)^{\alpha}$ for some $\alpha \in \mathbb{R}$. Since $d_{0}>0$, equations (21) and (22) imply $\lim _{n \rightarrow 0}\left|\lambda_{n+1}-\lambda_{n}\right|=\infty$ and hence, (20) holds.

When $H$ is $\mathcal{P} \mathcal{T}$-symmetric, the eigenvalues either are real or else appear in complex conjugates. Thus, if $H$ is $\mathcal{P} \mathcal{T}$-symmetric, then by monotonicity of magnitude of the large eigenvalues (20), $H$ cannot have an infinite pairs of complex conjugate eigenvalues and hence all but finitely many eigenvalues are real.

Next, we will prove Theorem 2 on a necessary and sufficient condition for an anharmonic oscillator $H$ having infinitely many real eigenvalues.

Proof of Theorem 2. Suppose that $H$ or its translation is $\mathcal{P} \mathcal{T}$-symmetric. It is easy to check that the potentials $V(z)$ and $V\left(z-z_{0}\right)$ generate the same set of eigenvalues for any $z_{0} \in \mathbb{C}$. Thus, since $H$ or its translation is $\mathcal{P} \mathcal{T}$-symmetric, eigenvalues of $H$ either are real or come in complex conjugates. Then by (20), we conclude that all but finitely many eigenvalues are real. Since there are infinitely many eigenvalues [12] as we noted in the Introduction, there are infinitely many real eigenvalues.

Suppose that $H$ has infinitely many real eigenvalues. Then first, from the properties of $K_{m, j}(a)$ in Remark 1 and (18), one can derive the following properties of $c_{j}(a)$ :

(i) The $c_{j}(a)$ are all real-valued polynomials in terms of the coefficients $a$ of $P$.

(ii) For $2 \leq j \leq m$, the polynomial $c_{j}(a)$ depends only on $a_{1}, a_{2}, \ldots, a_{j}$. Furthermore, it is a non-constant linear function of $a_{j}$.

Notice that if $c_{j}(a) \notin \mathbb{R}$ for some $0 \leq j \leq m+1$, then (3) implies that all but finitely many eigenvalues are non-real. Thus, if $H$ has infinitely many real eigenvalues, then $c_{j}(a) \in \mathbb{R}$ for all $0 \leq j \leq m+1$.

Recall that $c_{0}(a)$ is real and depends only on $m$, and $c_{1}(a)=0$. Next, if $a_{1} \neq 0$, then we can choose $z_{0}=-\frac{a_{1}}{m} i$ and we consider the translation of $H$, replacing the potential $V(z)$ by $V\left(z-z_{0}\right)$. The translated $H$ has its potential with a vanishing $z^{m-1}$-term (i.e., $\left.a_{1}=0\right)$. Then by the above properties $(i)$ and $(i i)$ of $c_{2}(a), c_{2}(a) \in \mathbb{R}$ is a real polynomial in $a_{1}$ and $a_{2}$, and it is a nonconstant linear function in $a_{2}$. Thus, since $a_{1}=0 \in \mathbb{R}$ and $c_{2}(a) \in \mathbb{R}$, we obtain $a_{2} \in \mathbb{R}$. Likewise, since $a_{1}, a_{2}, c_{3}(a) \in \mathbb{R}$, by the above properties of $c_{3}(a), a_{3} \in \mathbb{R}$ and hence, recursively we conclude that $a \in \mathbb{R}^{m}$. Therefore, the translated $H$ is $\mathcal{P} \mathcal{T}$-symmetric. 


\section{References}

[1] Bender C.M., Boettcher S., Real spectra in non-Hermitian Hamiltonians having $\mathcal{P} \mathcal{T}$-symmetry, Phys. Rev. Lett. 80 (1998), 5243-5246, physics/9712001.

[2] Bender C.M., Hook D.W., Mead L.R., Conjecture on the analyticity of $\mathcal{P} \mathcal{T}$-symmetric potentials and the reality of their spectra, J. Phys. A: Math. Theor. 41 (2008), 392005, 9 pages, arXiv:0807.0424.

[3] Caliceti E., Graffi S., Sjöstrand J., $\mathcal{P} \mathcal{T}$ symmetric non-self-adjoint operators, diagonalizable and nondiagonalizable, with a real discrete spectrum, J. Phys. A: Math. Theor. 40 (2007), 10155-10170, arXiv:0705.4218.

[4] Conway J.B., Functions of one complex variable. I, 2nd ed., Springer-Verlag, New York, 1995.

[5] Dorey P., Dunning C., Tateo R., Spectral equivalences, Bethe ansatz equations, and reality properties in $\mathcal{P} \mathcal{T}$-symmetric quantum mechanics, J. Phys. A: Math. Gen. 34 (2001), 5679-5704, hep-th/0103051.

[6] Hille E., Lectures on ordinary differential equations, Addison-Wesley Publ. Co., Reading, Massachusetts, 1969.

[7] Lévai G., Siegl P., Znojil M., Scattering in the $\mathcal{P} \mathcal{T}$-symmetric Coulomb potential, J. Phys. A: Math. Theor. 42 (2009), 295201, 9 pages, arXiv:0906.2092.

[8] Mostafazadeh A., Pseudo-Hermiticity versus $\mathcal{P} \mathcal{T}$ symmetry: the necessary condition for the reality of the spectrum of a non-Hermitian Hamiltonian, J. Math. Phys. 43 (2002), 205-214, math-ph/0107001.

[9] Shin K.C., On the reality of the eigenvalues for a class of $\mathcal{P} \mathcal{T}$-symmetric oscillators, Comm. Math. Phys. 229 (2002), 543-564, math-ph/0201013.

[10] Shin K.C., Eigenvalues of $\mathcal{P} \mathcal{T}$-symmetric oscillators with polynomial potentials, J. Phys. A: Math. Gen. 38 (2005), 6147-6166, math.SP/0407018.

[11] Shin K.C., Asymptotics of eigenvalues of non-self adjoint Schrödinger operators on a half-line, Comput. Methods Funct. Theory, to appear, arXiv:1001.5120.

[12] Sibuya Y., Global theory of a second order linear ordinary differential equation with a polynomial coefficient, North-Holland Mathematics Studies, Vol. 18, North-Holland Publishing Co., Amsterdam - Oxford, 1975.

[13] Znojil M., Siegl P., Lévai G., Asymptotically vanishing $\mathcal{P} \mathcal{T}$-symmetric potentials and negative-mass Schrödinger equations, Phys. Lett. A 373 (2009), 1921-1924, arXiv:0903.5468. 